\title{
Magnetohydrodynamics with chiral anomaly: Phases of collective excitations and instabilities
}

\author{
Koichi Hattori, ${ }^{1}$ Yuji Hirono, ${ }^{2}$ Ho-Ung Yee, ${ }^{3}$ and Yi Yin $\odot^{4}$ \\ ${ }^{1}$ Physics Department and Center for Particle Physics and Field Theory, \\ Fudan University, Shanghai 200433, China \\ ${ }^{2}$ Department of Physics, Brookhaven National Laboratory, Upton, New York 11973-5000, USA \\ ${ }^{3}$ Department of Physics, University of Illinois, Chicago, Illinois 60607, USA \\ ${ }^{4}$ Center for Theoretical Physics, Massachusetts Institute of Technology, \\ Cambridge, Massachusetts 02139, USA
}

(Received 13 January 2018; revised manuscript received 4 January 2019; published 26 September 2019)

\begin{abstract}
We study relativistic hydrodynamics with chiral anomaly and dynamical electromagnetic fields, namely chiral magnetohydrodynamics (CMHD). We formulate CMHD as a low-energy effective theory based on a generalized derivative expansion. We demonstrate that the modification of ordinary magnetohydrodynamics (MHD) due to chiral anomaly can be obtained from the second law of thermodynamics and is tied to the chiral magnetic effect. We further study the real-time properties of a chiral fluid by solving linearized CMHD equations. We discover a remarkable "transition" at an intermediate axial chemical potential $\mu_{A}$ between a stable chiral fluid at low $\mu_{A}$ and an unstable chiral fluid at high $\mu_{A}$. We summarize this transition in a "phase diagram" in terms of $\mu_{A}$ and the angle of the wave vector relative to the magnetic field. In the unstable regime, four collective modes carry both magnetic and fluid helicity, in contrary to MHD waves, which are unpolarized. Half of the helical modes grow exponentially in time, indicating the instability, while the other half become dissipative.
\end{abstract}

DOI: 10.1103/PhysRevD.100.065023

\section{INTRODUCTION}

Hydrodynamics is a versatile theory describing the realtime dynamics of a given interacting many-body system in the long-time limit [1]. In this limit, most degrees of freedom become irrelevant, since they relax within short timescales. The surviving dynamical variables are typically those related to the conservation laws. For instance, hydrodynamic variables for a normal fluid include the energy density $\epsilon$ and the fluid velocity $u^{\mu}$, which correspond to the conservation of energy and of momentum, respectively. A more complicated example is provided by a conducting fluid which is described by magnetohydrodynamics (MHD), the theory of which couples the hydrodynamic motion of the fluid to Maxwell's theory of electromagnetism. The dynamical variables of MHD include not only $\epsilon$ and $u^{\mu}$, but the magnetic field $B^{\mu}$ as well. Here, the field strength tensor and its dual are $F_{\mu \nu}$ and $\tilde{F}^{\mu \nu}=$ $\frac{1}{2} \epsilon^{\mu \nu \alpha \beta} F_{\alpha \beta}=B^{\mu} u^{\nu}-B^{\nu} u^{\mu}+\epsilon^{\mu \nu \alpha \rho} u_{\alpha} E_{\rho}$, respectively. The electric charge density $n$ and electric field $E^{\mu}$ are damped

Published by the American Physical Society under the terms of the Creative Commons Attribution 4.0 International license. Further distribution of this work must maintain attribution to the author(s) and the published article's title, journal citation, and DOI. Funded by SCOAP ${ }^{3}$. out at a rate proportional to the electric conductivity $\sigma$, and therefore should not be included as MHD variables (see Refs. [2-4] and the Appendix B). For this reason, we will consider a chiral fluid which is (vector) charge neutral throughout.

The primary purpose of this paper is to study the properties of chiral matter (systems involving chiral fermions) in the long-time limit. Chiral matter exhibits many interesting phenomena, some of which are closely tied to chiral anomaly. We wish to present a hydrodynamic approach for conducting chiral fluid by coupling the dynamics of axial (chiral) charge density $n_{A}$ to MHD, and we refer to the resulting theory as chiral MHD (CMHD); see Refs. [5-8] for previous studies. This theory would allow us to study those anomaly-induced effects which are absent in ordinary MHD. The places where such a theory can potentially be applied include the quark-gluon plasma (QGP) created by heavy-ion collisions $[9,10]$, newly discovered Dirac and Weyl semimetals [11,12], and the electroweak plasma produced in the primordial Universe after the big bang [13,14].

However, $n_{A}$ is distinguished from standard MHD variables because of their difference in the long-wavelength behavior of the relaxation rate. Specifically, $\Gamma_{A}$, the relaxation rate of $n_{A}$, is finite in the small wave vector (or gradient) $k$ limit, since axial current $J_{A}^{\mu}$ is not conserved 
due to quantum anomaly. Therefore, one has to identify an additional small parameter to make $\Gamma_{A}$ parametrically small so that $n_{A}$ can be counted as a parametrically slow mode (see Ref. [15] for a discussion on the extension of hydrodynamics with parametrically slow modes in generic situations). We identify this additional small parameter as the anomaly coefficient $C_{A}$ [see Eq. (3) below], and we will work in the limit $C_{A} \ll 1$. This identification is natural because $C_{A}$ tracks the effects of quantum anomaly, which are typically suppressed by the additional power of $\hbar$. Without such anomalous effects, $n_{A}$ would be conserved, and hence $\Gamma_{A}$ would vanish in the $C_{A} \rightarrow 0$ limit. In fact, we shall see $\Gamma_{A} \propto C_{A}^{2}$, which is analogous to the relaxation rate of standard MHD variables, which is proportional to $k^{2}$.

The very presence of this additional small parameter $C_{A}$ also necessitates the generalization of the standard procedure of derivative expansion for hydrodynamics to construct CMHD. We will formulate CMHD based on the double expansion in terms of the gradient number $k$ (times the mean free path $l_{\text {map }}$ ) and $C_{A}$ in Sec. II. By double expansion, we mean that both $C_{A}$ and $k l_{\text {mfp }}$ are small, but we do not assume any hierarchy between them. Here and hereafter, let us use $\mathcal{O}(\delta)$ to denote terms of the order $\mathcal{O}(k)$ ( $k$ times the mean free path) and/or $\mathcal{O}\left(C_{A}\right)$. As detailed below, we will express the stress-energy tensor $T^{\mu \nu}, J_{A}^{\mu}$, and $E^{\mu}$ in terms of the CMHD variables $\epsilon, u^{\mu}, B^{\mu}, n_{A}$ up to the first order in $\mathcal{O}(\delta)$. From now on, quantities at $\mathcal{O}(\delta)$ are sometimes labeled with the subscript (1). In our derivation, the stringent constraint imposed by the second law of thermodynamics is taken into account. The result of doing so yields

$$
E_{(1)}^{\mu}=-\frac{1}{\sigma}\left[C_{\mathrm{A}} \mu_{\mathrm{A}} B^{\mu}+\beta^{-1} \epsilon^{\mu \nu \alpha \rho} u_{\nu} \partial_{\alpha}\left(\beta H_{\rho}\right)\right] .
$$

In Eq. (1), $\beta$ is the inverse of the temperature and $H^{\mu}$ is the in-medium magnetic field. While the $\mathcal{O}(k)$ term in Eq. (1) already shows up in MHD, the presence of an $\mathcal{O}\left(C_{A}\right)$ term there, being proportional to the axial chemical potential $\mu_{A}$, is the distinctive feature of CMHD. We shall see that this term is closely related to the chiral magnetic effect (CME) [16-18] (see Refs. [10,19-22] for a recent review), the generation of electric current by a magnetic field in chiral matter with nonzero $n_{A}$.

We next explore the real-time properties of a chiral fluid by solving linearized CMHD, and we study the corresponding collective excitations in Sec. III. Our chief observation is the "transition" at an intermediate $\mu_{A}$ between a stable chiral fluid at low $\mu_{A}$ and an unstable chiral fluid at high $\mu_{A}$. In the unstable regime, there will be four collective modes carrying both magnetic and fluid helicity, in contrary to waves in MHD, which are unpolarized. Half of the helical modes have positive imaginary components, indicating the instability. The formulation of CMHD based on a new derivative expansion scheme together with the discovery of a qualitative difference in the dynamical properties of chiral fluid are the main findings of this paper.

\section{CHIRAL MHD}

The equations of motion for the CMHD variables $\epsilon, u^{\mu}$, $B^{\mu}$, and $n_{\mathrm{A}}$ consist of the energy-momentum conservation, the Bianchi identity, and the anomaly equation (we use the "most minus sign" convention for the metric $g^{\mu \nu}$ ):

$$
\begin{gathered}
\partial_{\mu} T^{\mu \nu}=0, \\
\partial_{\mu} \tilde{F}^{\mu \nu}=0, \\
\partial_{\mu} J_{\mathrm{A}}^{\mu}=-C_{\mathrm{A}} E \cdot B,
\end{gathered}
$$

where $J_{\mathrm{A}}^{\mu}$ is the axial current, and the anomaly coefficient is given by

$$
C_{A}=\frac{e^{2}}{2 \pi^{2}}
$$

Here, $T^{\mu \nu}$ is the energy-momentum tensor of the total system (i.e., the fluid and EM fields) so that it is conserved. Equation (2c) shows that the evolution of $n_{A}$ is closely related to the dynamics of EM fields. Indeed, the reconnection of magnetic flux could induce the change of $n_{A}$ [23]. Depending on the microscopic details of the systems under study, there could be other processes which contribute to the relaxation of $n_{A}$. For example, topological sphaleron transitions can also change the axial charge in a quark-gluon plasma (QGP), but the transition rate scales with $g^{10}$ for weakly coupled QGP. We have neglected those additional processes when writing down Eq. (2c) to simplify the discussion, and we leave their extension to future work.

At zeroth order $\left[\mathcal{O}\left(\delta^{0}\right)\right]$, the form of $T^{\mu \nu}, \tilde{F}^{\mu \nu}$ is identical to those of MHD. Therefore,

$$
\begin{gathered}
T^{\mu \nu}=\epsilon u^{\mu} u^{\nu}-p\left(g^{\mu \nu}-u^{\mu} u^{\nu}\right)-H^{\mu} B^{\nu}+T_{(1)}^{\mu \nu}+\mathcal{O}\left(\delta^{2}\right), \\
\tilde{F}^{\mu \nu}=B^{\mu} u^{\nu}-B^{\nu} u^{\mu}+\epsilon^{\mu \nu \alpha \rho} u_{\alpha} E_{\rho(1)}+\mathcal{O}\left(\delta^{2}\right), \\
J_{\mathrm{A}}^{\mu}=n_{\mathrm{A}} u^{\mu}+J_{\mathrm{A}(1)}^{\mu}+\mathcal{O}\left(\delta^{2}\right) ;
\end{gathered}
$$

see also Appendix A for a detailed discussion. Here, the inmedium magnetic field $H^{\mu}$ is conjugate to $B_{\mu}$, i.e.,

$$
d s=\beta d \epsilon-\left(\beta \mu_{\mathrm{A}}\right) d n_{\mathrm{A}}+\beta H_{\mu} d B^{\mu} .
$$

The entropy density $s$ and pressure $p$ are related by the thermodynamic relation:

$$
p=-\epsilon+\mu_{\mathrm{A}} n_{\mathrm{A}}+\beta^{-1} s-H \cdot B .
$$


We remind the reader that $T^{\mu \nu}$ refers to the total stress tensor (i.e., the sum of the fluid and Maxwell stress tensors) of the system. Therefore, there is a $B^{\mu} H^{\nu}$ term in Eq. (4a), and a dependence of $p$ on $B$. They originate from the Lorentz force that the charged fluid would experience (cf. Ref. [24]).

We next consider the entropy current $S^{\mu}=s u^{\mu}+s_{(1)}^{\mu}$ and require the positivity of the entropy production $\partial_{\mu} S^{\mu} \geq 0$. Transforming $\beta u_{\nu} \partial_{\mu} T^{\mu \nu}+\beta \mu_{A} \partial_{\mu} J^{\mu}+\beta H_{\mu} \partial_{\nu} \tilde{F}^{\mu \nu}$ using Eq. (2), we find (in the Landau fluid frame $\left.u_{\mu} T_{(1)}^{\mu \nu}=0\right)$

$$
\begin{aligned}
& \beta\left(\partial_{\mu} u_{\nu}\right) T_{(1)}^{\mu \nu}+\left[-\partial_{\mu}\left(\beta \mu_{A}\right) J_{A(1)}^{\mu}\right] \\
& \quad+E_{\mu(1)}\left[C_{\mathrm{A}} \mu_{\mathrm{A}} B^{\mu}+\beta^{-1} \epsilon^{\mu \nu \alpha \rho} u_{\nu} \partial_{\alpha}\left(\beta H_{\rho}\right)\right] \geq 0,
\end{aligned}
$$

with $S_{(1)}$ given by (A19); see Appendix A for more details. We note that $\partial_{\mu}\left(s u^{\mu}\right)=0$ (cf. Sec. 1 of Appendix A), meaning there is no entropy production for zeroth-order (i.e., ideal) CMHD. In order to satisfy the condition (7), it is sufficient to require each of the three terms on the lhs of Eq. (7) to be positive definite [cf. Eq. (A20)]. The expression for $T_{(1)}^{\mu \nu}$ in MHD has been determined previously $[2-4,25,26]$ and satisfies $\left(\partial_{\mu} u_{\nu}\right) T_{(1)}^{\mu \nu} \geq 0$. In other words, the constitutive relation for $T_{(1)}^{\mu \nu}$ is identical to that of MHD. Meanwhile, $\left[-\partial_{\mu}\left(\beta \mu_{A}\right) J_{A(1)}^{\mu}\right] \geq 0$ will be satisfied if $J_{A}^{\mu}=\lambda_{A} \partial^{\mu}\left(\beta \mu_{A}\right)$, where $\lambda_{A}$ is a positive transport coefficient. We finally turn to the condition

$$
E_{\mu(1)}\left[C_{\mathrm{A}} \mu_{\mathrm{A}} B^{\mu}+\beta^{-1} \epsilon^{\mu \nu \alpha \rho} u_{\nu} \partial_{\alpha}\left(\beta H_{\rho}\right)\right] \geq 0,
$$

which requires $E^{\mu}$ to be of the form given by Eq. (1). Here, a positive constant $\sigma$ will be identified with the electric conductivity shortly. For simplicity, we assume that $\sigma$ is isotropic, which is the case in a weak-magnetic-field limit. Nevertheless, our conclusion on the CME current holds even with a general tensor structure of $\sigma$, as is shown in Sec. 2 of Appendix A.

We now demonstrate that the first term on the rhs of Eq. (1) is tied to CME. To simplify our analysis, we take $\beta$ to be homogeneous and rewrite the spatial part of Eq. (1) in the local rest frame of fluid:

$$
\boldsymbol{E}=\sigma^{-1}\left(-C_{A} \mu_{A} \boldsymbol{B}+\nabla \times \boldsymbol{B}\right) .
$$

Employing Ampere's law $\boldsymbol{j}=\nabla \times \boldsymbol{B}$, we have

$$
\boldsymbol{j}=C_{A} \mu_{A} \boldsymbol{B}+\sigma \boldsymbol{E} .
$$

Two implications follow from Eq. (10). First, $\sigma$ in Eq. (1) has to be the conductivity so that $\sigma \boldsymbol{E}$ is the usual $\mathrm{Ohm}$ current. Second, we now recover the CME current from Eq. (1). Notice that the dynamical variables as well as the counting scheme here are different from those in earlier works. For example, $E^{\mu}, B^{\mu}$ are nondynamical, and are counted as $\mathcal{O}(k)$ in Ref. [27]. Despite these differences, we find the same form of the CME current, which exemplifies the universal nature of CME. To the best of our knowledge, the demonstration of this universality within the framework of CMHD based on the second law of thermodynamic is new in literature. In fact, there are studies which show that the form of other anomaly-induced effects, in particular those of chiral vortical effect, is nonuniversal with a dynamical gauge field [28]. In this regard, our result on the universal form of CME contribution is quite remarkable.

We wish to reiterate the novelty and necessity of double expansion in terms of $C_{A}$ and gradient in CHMD, through which we obtain Eq. (1) and hence the manifestation of CME in Eqs. (1) and (10). If we were using the conventional gradient expansion, then the first term, i.e., the CME term, in Eq. (1) has to be counted as zeroth order in the gradient. This contradicts with the assumption that the electric field $E^{\mu}$ is not a slow variable and cannot be counted as the zeroth-order term in the expansion. Generally speaking, the very existence of nonhydrodynamic slow modes suggests the presence of at least one additional small parameter which controls the slowness of such modes in the system of interest. For the case of chiral fluid with slow evolving $n_{A}$, we identify this slow parameter as $C_{A}$. Indeed, let us recover $\hbar$ dependence of the chiral magnetic conductivity $C_{A} \mu_{A}$ [29], which is of the order $\mu_{A} / \hbar^{2}$ (cf. Ref. [30]), and that of the Ohm conductivity $\sigma$, which is of the order $T / g^{4}$, where $g$ generically denotes the coupling constant. Following Ref. [31], we will require that $g / \hbar$ approach a constant in the $\hbar \rightarrow 0$ limit. Doing so would make the loop expansion the same as the expansion in powers of $\hbar$. Consequently, $\sigma \sim 1 / \hbar^{4}$, and the CME term in Eq. (10) is parametrically smaller than the Ohmic current term in this limit. This in turn confirms that $C_{A}$ tracks the quantum effect of chiral anomaly, which is suppressed by the additional power of $\hbar$, as we claimed earlier.

\section{COLLECTIVE MODES}

We now consider fluctuations around a uniform static background: $\epsilon(t, \boldsymbol{x})=\epsilon+\delta \epsilon(t, \boldsymbol{x}), u^{\mu}(t, \boldsymbol{x})=(1, \boldsymbol{v}(t, \boldsymbol{x}))$, $B^{\mu}(t, \boldsymbol{x})=(-\boldsymbol{B} \cdot \boldsymbol{v}(t, \boldsymbol{x}),-\boldsymbol{B}+\delta \boldsymbol{B}(t, \boldsymbol{x}))$, and $n_{A}(t, \boldsymbol{x})=$ $n_{A}+\delta n_{A}(t, \boldsymbol{x})$. Our formulation of CMHD is valid as long as both $C_{A}$ and the gradient are small. In what follows, we will solve linearized CMHD for those fluctuations in frequency $\omega$ and wave vector $\boldsymbol{k}$ space by specifying the following hierarchy among $k$ and other scales. First, our focus will be on the regime $k \ll C_{A} \mu_{A}$, since early studies indicate that chiral plasma would become unstable in this momentum regime. Consequently, we neglect the second term on the rhs of Eq. (1). In this limit, $\partial_{t} n_{A}=C_{A} \boldsymbol{E} \cdot \boldsymbol{B}$ will become a relaxation equation of $n_{A}$ upon substituting $\boldsymbol{E}=-C_{A} \mu_{A} \boldsymbol{B} / \sigma$. The corresponding relaxation rate $\Gamma_{A} \sim C_{A}^{2}$, as we advertised earlier. Next, we will require 
$\omega(k) \gg \Gamma_{A} \sim C_{A}^{2}$ so that the evolution of $\delta n_{A}(t, \boldsymbol{x})$ is decoupled from other fluctuating variables. In the small- $C_{A}$ limit, there is indeed a wide range of $k$ satisfying both conditions. Finally, we assume $\eta k, \zeta k \ll \epsilon$ as in ordinary hydrodynamics, where $\eta$ and $\zeta$ are the shear and bulk viscosity, respectively. For this reason, we will not include the contribution due to $T_{(1)}^{\mu \nu}$ from now on.

To proceed, we will use a simplified equation of state $p=p_{f}\left(\epsilon_{f}\right)-\mathcal{B}^{2} / 2$ so that $H^{\mu}=B^{\mu}$. Here $\epsilon_{f}=\epsilon-\mathcal{B}^{2} / 2$ and $p_{f}$ are the fluid parts of the energy density and pressure, respectively, where we have defined

$$
\mathcal{B} \equiv \sqrt{-B \cdot B} .
$$

For definiteness, we will use $p_{f}\left(\epsilon_{f}\right)=c_{s}^{2} \epsilon_{f}$, where $c_{s}$ is the sound velocity. The linearized equations for (rescaled) fluctuation fields,

$$
\delta \tilde{\epsilon}_{f} \equiv \frac{\delta \epsilon_{f}}{\left(e_{f}+p_{f}\right)}, \quad \boldsymbol{b} \equiv \frac{\delta \boldsymbol{B}}{\sqrt{\epsilon_{f}+p_{f}}},
$$

now read

$$
\begin{gathered}
i \omega \delta \tilde{\epsilon}_{f}=-i k v_{L}, \\
i \omega v_{L}=-i k\left(c_{s}^{2} \delta \tilde{\epsilon}_{f}-u_{A} \sin \theta b_{2}\right), \\
i \omega v_{1}=i k u_{A} \cos \theta b_{1}, \quad i \omega v_{2}=i k u_{A} \cos \theta b_{2}, \\
i \omega b_{1}=i k\left(u_{A} \cos \theta v_{1}+\epsilon_{A} b_{2}\right), \\
i \omega b_{2}=i k\left[u_{A}\left(\sin \theta v_{L}+\cos \theta v_{2}\right)-\epsilon_{A} b_{1}\right],
\end{gathered}
$$

where $\theta$ is the relative angle between $\boldsymbol{k}$ and $\boldsymbol{B}$. By introducing a standard orthogonal unit basis $\hat{e}_{1} \propto \hat{B} \times \hat{k}$, $\hat{e}_{2}=\hat{k} \times \hat{e}_{1}$ and $\hat{k}$ [32], we have decomposed the fluctuation fields as $\boldsymbol{v}=v_{1} \hat{e}_{1}+v_{2} \hat{e}_{2}+v_{L} \hat{k}$ and $\boldsymbol{b}=b_{1} \hat{e}_{1}+b_{2} \hat{e}_{2}$ (note that $\nabla \cdot \boldsymbol{b}=0$ ). For later convenience, we have introduced two important dimensionless parameters: namely, Alfvén velocity $u_{\mathrm{A}} \equiv B / \sqrt{\epsilon_{f}+p_{f}}$ and $\epsilon_{A}=C_{A} \mu_{A} / \sigma$. We have further assumed $u_{A} \ll 1$, and have dropped terms suppressed $u_{\mathrm{A}}^{2} \ll 1$ when writing down Eq. (13). However, $\epsilon_{\mathrm{A}} / u_{\mathrm{A}}$ can be $\mathcal{O}(1)$, since $\epsilon_{\mathrm{A}} \propto C_{A} \ll 1$.

The dispersion relation $\omega(k)=V k$ of collective modes can be determined by solving Eq. (13), where "group velocity" $V$ satisfies

$$
\begin{aligned}
& \left(V^{2}-u_{A}^{2} \cos ^{2} \theta\right)\left[V^{4}-\left(u_{A}^{2}+c_{\mathrm{s}}^{2}\right) V^{2}+c_{\mathrm{s}}^{2} u_{\mathrm{A}}^{2} \cos ^{2} \theta\right] \\
& \quad+\epsilon_{\mathrm{A}}^{2} V^{2}\left(V^{2}-c_{\mathrm{s}}^{2}\right)=0 .
\end{aligned}
$$

Equation (14) has six roots, corresponding to six collective modes. Furthermore, by expressing $v_{1,2}$ in terms of $b_{1,2}$ using Eq. (13c), and substituting the resulting expressions into Eq. (13d), we found

$$
\frac{v_{1}}{v_{2}}=\frac{b_{1}}{b_{2}}=\frac{\epsilon_{\mathrm{A}} V}{u_{\mathrm{A}}^{2} \cos ^{2} \theta-V^{2}} .
$$

Equation (15) is very informative in at least two aspects. First, it implies that the relative phase between $b_{1}, b_{2}$ is the same as that between $v_{1}, v_{2}$. If a collective mode carries a positive (negative) magnetic helicity, it also carries a fluid helicity of the same chirality. Such a mode will be called the RH (LH) mode below. By definition, magnetic and fluid helicity are positive (negative) if $\delta \boldsymbol{A} \cdot \delta \boldsymbol{B}>0(<0)$ and $(\nabla \times \boldsymbol{v}) \cdot \boldsymbol{v}>0(<0)$, respectively. Here $\delta \boldsymbol{A}$ is the vector gauge potential satisfying $\boldsymbol{B}=\nabla \times \delta \boldsymbol{A}$. Second, Eq. (15) will tell us the polarization of each collective mode with a given $V$. For instance, Eq. (15) implies that a mode with a real-valued $V$ is linearly polarized, whereas that with a purely imaginary $V$ is circularly polarized.

Let us first consider Eq. (13) in two limiting cases. In the limit $\epsilon_{\mathrm{A}} / u_{A}=0$, Eq. (13) is reduced to the linearized MHD. The collective modes are well known as the Alfvén wave and the fast and slow magnetosonic waves [33] with group velocities given by $V_{A}, V_{F}$, and $V_{S}$, respectively. Solving Eq. (14) at $\epsilon_{A}=0$, one finds

$$
\begin{gathered}
V_{A}^{2}=u_{A}^{2} \cos ^{2} \theta, \\
V_{F, S}^{2}=\frac{\left(u_{A}^{2}+c_{s}^{2}\right) \pm \sqrt{u_{A}^{4}+c_{s}^{4}-2 u_{A}^{2} c_{s}^{2} \cos (2 \theta)}}{2},
\end{gathered}
$$

where a $+(-)$ sign corresponds to $V_{F}\left(V_{S}\right)$. We note that $V_{F, S}$ and $V_{A}$ are real, indicating that MHD waves are unpolarized and ordinary MHD systems are stable.

Now we turn to the opposite limit, $\epsilon_{\mathrm{A}} / u_{A} \gg 1$. In this case, the evolution of $b_{1}$ and $b_{2}$ is decoupled from that of $v$ and $\delta \tilde{e}_{f}$, and is described by setting $u_{A}=0$ in Eqs. (13d) and $(13 \mathrm{e})$. The corresponding collective modes are $\left(b_{1}, b_{2}\right) \propto(1, \pm i)$, corresponding to circularly polarized (helical) magnetic fields, with purely imaginary $V= \pm i \epsilon_{A}$. Such modes are referred to in literature as Chern-Simon (CS) modes [34]. Half of the CS modes have a positive imaginary part, signifying the instability of a chiral plasma, as discussed earlier in Refs. [14,34,35].

If we put the preceding analysis at small and large $\mu_{A}$ $\left(\epsilon_{A}\right)$ together, we conclude that a chiral fluid with a dynamical magnetic field must have at least one "transition" at an intermediate $\mu_{A}$ between a stable chiral fluid at low $\mu_{A}$ and a unstable chiral fluid at high $\mu_{A}$. We now put this qualitative expectation on a quantitative basis by computing the $V$ of each collective mode at a given $\epsilon_{\mathrm{A}} / u_{A}$ and $\theta$ by solving Eq. (14). It is sufficient to consider $0 \leq \theta \leq \pi / 2$, since $V$ determined from Eq. (14) will depend on $\cos ^{2}(\theta)$ only. In Fig. 1, we present a "phase diagram," which charts stable and unstable regimes ("phases") in the $\epsilon_{\mathrm{A}}-\theta$ plane.

In the low- $\mu_{A}$ "phase," i.e., "Phase I" (red regime), a chiral fluid is stable. All modes are akin to MHD waves up 


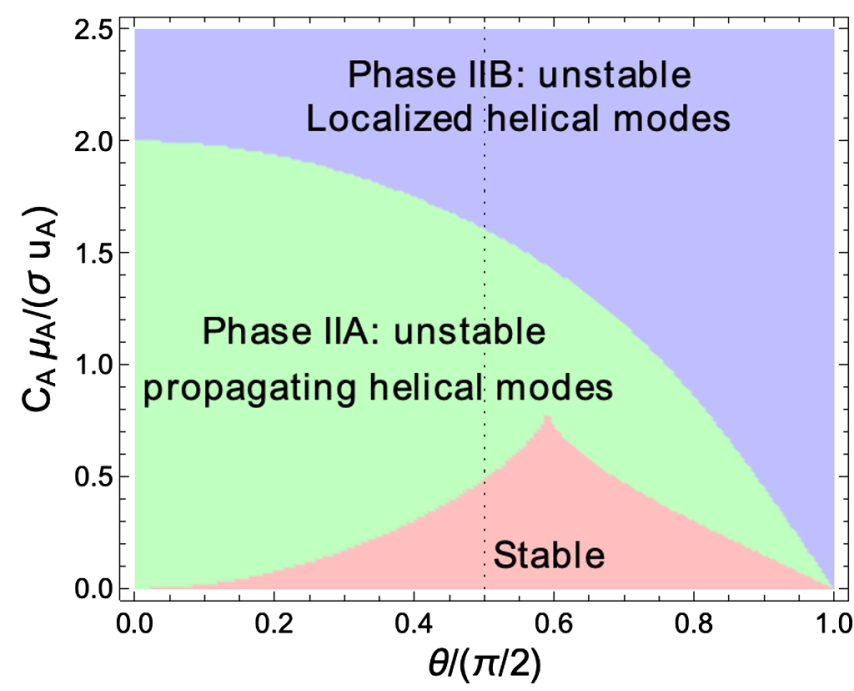

FIG. 1. A "phase diagram" charting the stable and unstable regimes of chiral fluid in the $\theta-\epsilon_{\mathrm{A}}$ plane (with $c_{\mathrm{s}} / u_{\mathrm{A}}=0.6$ ); see text. The dashed vertical (horizontal) curve represents the fixed value of $\theta\left(\epsilon_{\mathrm{A}}\right)$ used in Fig. 2.

to the modifications of the group velocities which are realvalued. However, as we increase $\epsilon_{A}$, the system transits to the unstable phase, i.e., "Phase II." While there are still two modes similar to ordinary MHD waves in this phase, the remaining four modes become helical and have nonzero $\operatorname{Im} V$. Half of those modes have $\operatorname{Im} V>0$, indicating their instability. As a specific example, Figs. 2(a) and 2(b) show the real and imaginary parts of $V$ for all six modes as functions of $\epsilon_{\mathrm{A}}$ at a fixed $\theta=\pi / 4$.

Figure 1 reveals that a chiral fluid is stable for a small but finite $\mu_{A}$ for any generic $\theta$. This is in stark contrast with the case of chiral plasma, which would become unstable in the presence of an infinitesimal small $\mu_{A}$ [14,34,35]. To understand this difference, let us consider, without losing generality, the modification of $V$ at small $\epsilon_{A}$ of the mode which corresponds to an Alfvén wave at $\epsilon_{A}=0$. Substituting $V=V_{A}+\Delta V$ into Eq. (14) and expanding it to linear order in $\Delta V$, we find

$$
\Delta V=-\frac{\epsilon_{A}^{2}\left(V_{A}^{2}-c_{S}^{2}\right) V_{A}}{2\left(V_{A}^{2}-V_{F}^{2}\right)\left(V_{A}^{2}-V_{S}^{2}\right)} .
$$

To obtain Eq. (18), we have used the fact that the first line of Eq. (14) can be put into to the form $\left(V^{2}-V_{F}^{2}\right)\left(V^{2}-V_{S}^{2}\right)\left(V^{2}-V_{A}^{2}\right)$. Equation (18) shows that $\Delta V$ remains real, i.e., $\operatorname{Im} V=0$, for sufficiently small $\epsilon_{A}$ and for $\theta$ at which there is no degeneracy among $V_{F, S}, V_{A}$, so that the denominator of Eq. (18) is nonzero. Consequently, chiral fluid is stable in this case, except for $\theta=0$ where $V_{F}=V_{A}=u_{A}$, and for $\theta=\pi / 2$ where $V_{S}=V_{A}=0$ [cf. Eqs. (16) and (17)]. Putting it in a perhaps more intuitive, albeit less rigorous way, we can think of those helical modes in the unstable phase as originating from a mixture of different linearly polarized MHD waves. Such a mixture will not be energetically favorable unless the group velocity of two different MHD waves becomes identical.

We now focus on those four modes which become helical in the unstable phase. One can show from Eq. (15) that those helical modes have the remarkable properties of "selective growth"- namely, when $\epsilon_{\mathrm{A}}>0\left(\epsilon_{\mathrm{A}}<0\right)$, the $\operatorname{Im} V$ of $\mathrm{RH}(\mathrm{LH})$ modes is positive, meaning that $\mathrm{RH}(\mathrm{LH})$ modes are "selected" to grow exponentially in time. CS modes for chiral plasma also exhibit the properties of selective growth, and its physical origin has been discussed in many early works (e.g., Refs. [14,34-36]). Since chiral anomaly can redistribute helicity between the fermionic and magnetic parts, the chiral plasma will tend to minimize the energy cost at a fixed helicity by populating modes with a definite helicity. The physics is similar here. What is distinctive about helical modes of chiral fluid is that they carry nonzero fluid helicity in addition to magnetic helicity, since the magnetic field and fluid field are coupled to each other.

To illustrate this close relationship between the chirality of the helicity and the instability of those collective modes, we plot the trajectories of the Stokes vector [32], $s=$ $\left(b_{1}^{2}-b_{2}^{2}, 2 \operatorname{Re}\left[b_{1} b_{2}^{*}\right], 2 \operatorname{Im}\left[b_{1} b_{2}^{*}\right]\right) /\left(b_{1}^{2}+b_{2}^{2}\right)$, corresponding to those four modes with varying $\epsilon_{A}$ at $\theta=\pi / 2$ on a unit

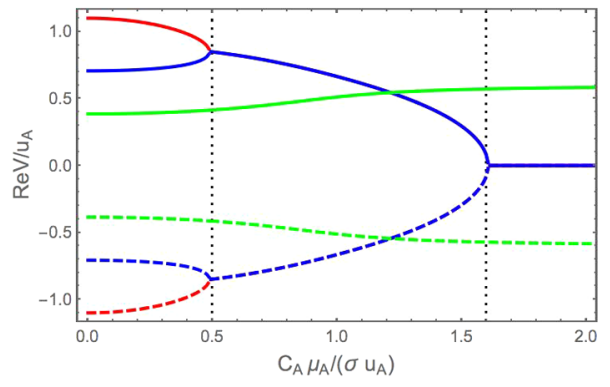

(a)

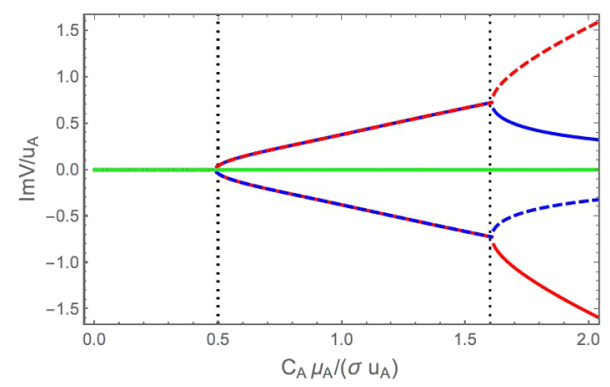

(b)

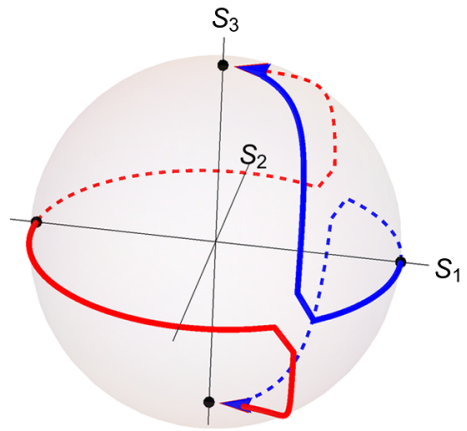

(c)

FIG. 2. Plots of (a) the real part and (b) the imaginary part of $V$, and (c) the polarizations vs $\epsilon_{\mathrm{A}} / u_{\mathrm{A}}$ at $\theta=\pi / 4$ and $c_{\mathrm{s}} / u_{\mathrm{A}}=0.6$; see the text for more. The corresponding modes are shown in the same colors from (a) to (c). Vertical lines show the phase boundaries in Fig. 1. A pair of modes corresponding to the green beaches in (a) and (b) always stay at the equator and are not shown in (c). 
sphere (the Poincaré sphere) in Fig. 2(c). By definition, a point on the equator of the Poincaré sphere specifies a linear polarization, while points on the upper and lower hemispheres indicate left-handed and right-handed polarization, respectively. In particular, the north and south poles correspond to the circular polarizations. The red and blue trajectories start at $s=(-1,0,0)$ and $s=(1,0,0)$ (at the equator of the Poincaré sphere), respectively, corresponding to $\epsilon_{A}=0$, and "flow" to the upper/lower hemispheres when the transition occurs from Phase I to II, and eventually approach the north/south poles. Notice the correspondences between the polarization states and the dispersion relations shown in the same colors from Figs. 2(a) to 2(c).

While in the high- $\mu_{A}$ "phase," i.e., "Phase IIB," the values of $V$ for helical CMHD modes are purely imaginary; similarly to CS modes in the chiral plasma, those four helical modes have complex-valued $V$ at an intermediate- $\mu_{A}$ regime ("Phase IIA"). We will call them "chiral magnetohelical modes (CMHMs)." It might be useful to view CMHMs as an outcome of an interesting hybridization of MHD waves and CS modes. They "inherit" the ability of propagation in space $(\operatorname{Re} V \neq 0)$ from MHD waves, and that of carrying magnetic helicity from CS modes. The presence of such new collective modes has not been reported in the preceding studies of CMHD [5-8]. CMHMs are also distinct from collective modes in chiral fluid with a nondynamical magnetic field [37-40]. The emergence of CMHMs clearly demonstrates the rich physics underlying CMHD.

\section{SUMMARY AND IMPLICATIONS}

We have presented a formulation of hydrodynamic theory for a chiral fluid with a dynamical magnetic field based on a generalization of derivative expansion. We derive the manifestation of CME in CHMD at the first order in this expansion scheme. It would be interesting to extend our formulation to a higher order to study chiral vortical effect and other anomaly-induced phenomena in CMHD (e.g., Ref. [41]). In addition, we explore the real-time properties of chiral fluid, and find a qualitative difference in the aspects of stability and polarization of collective modes.

In this work, we focus on the basic formulation and general properties of chiral fluid. Our findings can be applied to specific chiral matter, such as Weyl semimetal [42] and QGP created in heavy-ion collisions. As for the latter, the dynamics of baryon density can be potentially important, and it is particular relevant to the coming lowbeam-energy scan at RHIC. It would be interesting to extend the present analysis of CMHD by including $n_{B}$ as well.

\section{ACKNOWLEDGMENTS}

This work is partially supported by the U.S. Department of Energy, Office of Science, Office of Nuclear Physics, with Grants No. DE-SC0012704 (Y.H.), No. DESC0018209 (H.-U. Y.), and No. DE-SC0011090 (Y. Y.), and within the framework of the Beam Energy Scan Theory Topical Collaboration. K. H. is supported by the China Postdoctoral Science Foundation under Grants No. 2016M590312 and No. 2017T100266. We thank Paolo Glorioso, Xu-Guang Huang, Matthias Kaminski, Dam Son, and Naoki Yamamoto for insightful comments on the manuscript. We are grateful to anonymous referees for insightful suggestions which improve the manuscript significantly.

\section{APPENDIX A: THE CONSTITUTIVE RELATION FOR CHIRAL MHD}

In this Appendix, we will supplement the discussion in Sec. II with more details on the form of the constitutive relation of CMHD. We shall see that the second law of thermodynamics imposes an important constraint on such relation.

We would like to express $\left\{T^{\mu \nu}, J_{\mathrm{A}}^{\mu}, \tilde{F}^{\mu \nu}\right\}$ in terms of CMHD variables $\left\{\epsilon, B, n_{A}\right\}$ by generalizing the standard procedure of derivative expansion to the double expansion in terms of gradient and $C_{A}$ :

$T^{\mu \nu}=T_{(0)}^{\mu \nu}+T_{(1)}^{\mu \nu}, \quad \tilde{F}^{\mu \nu}=\tilde{F}_{(0)}^{\mu \nu}+\tilde{F}_{(1)}^{\mu \nu}, \quad J_{\mathrm{A}}^{\mu}=J_{\mathrm{A}(0)}^{\mu}+J_{\mathrm{A}(1)}^{\mu}$,

as we explained earlier. We will use the subscripts (0) and (1) to denote quantities at zeroth and first order in $\delta$, respectively. As a reminder, $\mathcal{O}(\delta)$ denotes quantities of the order $\mathcal{O}(k)$ and/or $\mathcal{O}\left(C_{A}\right)$. CMHD variables are counted as zeroth order in $\mathcal{O}(\delta)$. Non-CMHD variables are counted as first order or even a higher order in $\delta$. In particular, $E^{\mu}$ is counted as $\mathcal{O}(\delta)$.

At zeroth order in $\delta$, the (total) energy momentum tensor, the axial current, and $\tilde{F}^{\mu \nu}$ can be written in general as

$$
\begin{gathered}
T_{(0)}^{\mu \nu}=\epsilon u^{\mu} u^{\nu}-X \Delta^{\mu \nu}-Y B^{\mu} B^{\nu}, \\
\tilde{F}_{(0)}^{\mu \nu}=B^{\mu} u^{\nu}-B^{\nu} u^{\mu}, \\
J_{\mathrm{A}(0)}^{\mu}=n_{\mathrm{A}} u^{\mu},
\end{gathered}
$$

where

$$
\Delta^{\mu \nu}=g^{\mu \nu}-u^{\mu} u^{\nu} .
$$

Here, $X$, and $Y$ are functions of CMHD variables, as we shall determine shortly. A term proportional to $B^{\mu}$ is not allowed in $J_{\mathrm{A}(0)}^{\mu}$ based on the symmetry consideration. For such a term to be present, its prefactor has to be $C$-odd, since $B^{\mu}$ is $C$-odd and $J_{\mathrm{A}}^{\mu}$ is $C$-even. However, the electric charge density $n$ vanishes in the limit $\delta \rightarrow 0$, and so it is the 
prefactor of such terms. For the same reason, we do not include a term proportional to $\left(B^{\mu} u^{\nu}+B^{\nu} u^{\mu}\right)$ in $T_{(0)}^{\mu \nu}$. Note that the expression for $\tilde{F}_{(0)}^{\mu \nu}$ follows from the definition of $B^{\mu}$.

In what follows, we will obtain the expression for $X, Y$ and $T_{(1)}^{\mu \nu}, J_{\mathrm{A}(1)}^{\mu}, E_{(1)}^{\mu}$ via the second law of thermodynamics. Note that by definition $E_{(1)}^{\mu}$ is related to $\tilde{F}_{(1)}^{\mu \nu}$ as

$$
\tilde{F}_{(1)}^{\mu \nu}=\epsilon^{\mu \nu \alpha \beta} u_{\alpha} E_{(1) \beta} .
$$

For this purpose, we consider the entropy current $S^{\mu}$ :

$$
S^{\mu}=S_{(0)}^{\mu}+S_{(1)}^{\mu},
$$

where the zeroth-order entropy current is given by

$$
S_{(0)}^{\mu}=s u^{\mu} .
$$

The divergence of $S^{\mu}$ now reads

$$
\begin{aligned}
\partial_{\mu} S^{\mu} & =D s+s \theta+\partial_{\mu} S_{(1)}^{\mu} \\
& =\beta D \epsilon-\left(\beta \mu_{\mathrm{A}}\right) D n_{\mathrm{A}}+\beta H_{\mu} D B^{\mu}+s \theta+\partial_{\mu} S_{(1)}^{\mu},
\end{aligned}
$$

where from the first line to the second line we have used Eq. (5). Here we have introduced the short-handed notation:

$$
D \equiv u_{\mu} \partial^{\mu}, \quad \theta \equiv \partial_{\mu} u^{\mu}
$$

On the other hand, by substituting Eqs. (A2), (A4), and (A1) into Eq. (2), we have

$$
\begin{aligned}
& D \epsilon=-(\epsilon+X) \theta-Y B_{\nu}(B \cdot \partial) u^{\nu}+\left(\partial_{\mu} u_{\nu}\right) T_{(1)}^{\mu \nu}, \quad(\mathrm{A} 9 \mathrm{a}) \\
& D B^{\mu}=-B^{\mu} \theta+(B \cdot \partial) u^{\mu}+u^{\mu}(\partial \cdot B)+\partial_{\mu}\left(\epsilon^{\mu \nu \alpha \beta} u_{\alpha} E_{(1) \beta}\right),
\end{aligned}
$$

$$
D n_{A}=-n_{A} \theta-\partial_{\mu} J_{A(1)}^{\mu}-C_{\mathrm{A}} E_{(1)} \cdot B
$$

In Eq. (A9a), we have used the fact that $u_{\nu} T_{(1)}^{\mu \nu}=0$, since we are working in the Landau fluid frame throughout. Combining Eq. (A9) and Eq. (A7) yields

$$
\begin{aligned}
\partial_{\mu} S^{\mu}= & D s+s \theta+\partial_{\mu} s_{(1)}^{\mu} \\
= & \beta \theta\left(-X-\epsilon+s T+\mu_{\mathrm{A}} n_{\mathrm{A}}-H \cdot B\right) \\
& +\beta\left(H_{\mu}-Y B_{\mu}\right)(B \cdot \partial) u^{\mu} \\
& +\beta\left(\partial_{\mu} u_{\nu}\right) T_{(1)}^{\mu \nu}+\left(\beta \mu_{\mathrm{A}}\right)\left(\partial \cdot J_{\mathrm{A}(1)}+C_{\mathrm{A}} E_{(1)} \cdot B\right) \\
& +\beta H_{\mu} \partial_{\nu}\left(\epsilon^{\mu \nu \alpha \rho} u_{\alpha} E_{(1) \rho}\right)+\partial \cdot S_{(1)} .
\end{aligned}
$$

Equation (A10) will be the starting point of the analysis in the subsequent Secs. 1 and 2.

\section{The constitutive relation at zeroth order in $\delta$ : Ideal chiral MHD}

To determine $T_{(0)}^{\mu \nu}$, we consider Eq. (A10) at first order in $\delta$. By requiring that there be no entropy production at this order, we have

$$
\begin{aligned}
\partial \cdot S_{(0)}= & \beta \theta\left(-X-\epsilon+s T+\mu_{\mathrm{A}} n_{\mathrm{A}}-H \cdot B\right) \\
& -\beta\left(H_{\mu}-Y B_{\mu}\right)(B \cdot \partial) u^{\mu} \\
= & 0 .
\end{aligned}
$$

Therefore, $X, Y$ satisfy

$$
\begin{gathered}
H_{\mu}=Y B_{\mu}, \\
X=-\epsilon+s T+\mu_{\mathrm{A}} n_{\mathrm{A}}-H \cdot B,
\end{gathered}
$$

meaning that $Y B^{\mu}$ is the in-medium magnetic field $H^{\mu}$, and $X$ must be identified with pressure $p$ after comparing Eq. (A12b) with Eq. (6). Substituting Eq. (A12) into Eq. (A2), we obtain $T_{(0)}^{\mu \nu}$ used in Eq. (4):

$$
T_{(0)}^{\mu \nu}=\epsilon u^{\mu} u^{\nu}-p \Delta^{\mu \nu}-H^{\mu} B^{\nu} .
$$

While in a large body of literature on relativistic MHD, energy density and pressure are often divided into the fluid and EM parts (cf. Refs. [25,26]), in this work, $\epsilon$ and $p$ denote the total energy and pressure, respectively, including the contributions from both fluid and EM field. The reason for doing so is that making the above-mentioned division could be physically impossible if the constitution of the fluid is strongly coupled to the EM field. As commented by W. Israel in Ref. [24], "questions about which part should be called the 'electromagnetic energy tensor' are near semantics and to a large extent, superfluous." That said, we could recover the conventional constitutive relation of ideal MHD from Eq. (A13) as follows: To simplify the discussion, we will assume $H^{\mu}=B^{\mu}$ and decompose the total energy density and pressure in the following form:

$$
\epsilon=\epsilon_{f}+\frac{1}{2} \mathcal{B}^{2}, \quad p=p_{f}+\frac{1}{2} \mathcal{B}^{2},
$$

with $e_{f}$ and $p_{f}$ being the fluid parts and $\mathcal{B}=\sqrt{-B \cdot B}$. Then, the energy momentum tensor of the conventional ideal MHD [cf. Eqs. (3) and (4) in Ref. [26] ] is reproduced as

$$
T_{(0)}^{\mu \nu}=T_{(0) \text { fluid }}^{\mu \nu}+T_{(0) \mathrm{EM}}^{\mu \nu},
$$

where 


$$
\begin{gathered}
T_{(0) \text { fluid }}^{\mu \nu}=\epsilon_{f} u^{\mu} u^{\nu}-p_{f} \Delta^{\mu \nu}, \\
T_{(0) \mathrm{EM}}^{\mu \nu}=\mathcal{B}^{2} u^{\mu} u^{\nu}-\frac{1}{2} \mathcal{B}^{2} g^{\mu \nu}-B^{\mu} B^{\nu} .
\end{gathered}
$$

We note that $T_{(0) \mathrm{EM}}^{\mu \nu}$ is the conventional EM stress-energy tensor in the absence of electric field.

\section{The constitutive relation at first order in chiral MHD and CME}

To constrain the constitutive relation at $\mathcal{O}(\delta)$, we consider Eq. (A10) at second order in $\delta^{2}$. The entropy production has to be positive definite at this order. Therefore,

$$
\begin{aligned}
& \beta\left(\partial_{\mu} u_{\nu}\right) T_{(1)}^{\mu \nu}+\beta \mu_{\mathrm{A}}\left(\partial \cdot J_{\mathrm{A}(1)}+C_{\mathrm{A}} E_{(1)} \cdot B\right) \\
& \quad+\beta H_{\mu} \partial_{\nu}\left(\epsilon^{\mu \nu \alpha \beta} u_{\alpha} E_{(1) \beta}\right)+\partial \cdot S_{(1)} \\
& =\partial_{\mu}\left[S_{(1)}^{\mu}+\beta \mu_{\mathrm{A}} J_{\mathrm{A}(1)}^{\mu}-\epsilon^{\mu \nu \alpha \beta} \beta H_{\nu} u_{\alpha} E_{(1) \beta}\right] \\
& \quad+T_{(1)}^{\mu \nu} \partial_{\mu}\left(\beta u_{\nu}\right)-J_{\mathrm{A}(1)} \cdot \partial\left(\beta \mu_{\mathrm{A}}\right) \\
& \quad+E_{(1) \rho}\left[C_{\mathrm{A}} \beta \mu_{\mathrm{A}} B^{\rho}+\epsilon^{\mu \nu \alpha \rho} \partial_{\mu}\left(\beta H_{\nu}\right) u_{\alpha}\right], \\
& \geq 0 .
\end{aligned}
$$

We first identify the first-order entropy current as those terms in the bracket of Eq. (A18a):

$$
s_{(1)}^{\mu}=-\beta \mu_{\mathrm{A}} J_{\mathrm{A}(1)}^{\mu}+\epsilon^{\mu \nu \alpha \rho} \beta H_{\nu} u_{\alpha} E_{(1) \rho} .
$$

Furthermore, the following relations are sufficient to guarantee the positivity of entropy production:

$$
\begin{gathered}
T_{(1)}^{\mu \nu}\left(\partial_{\mu} u_{\nu}\right) \geq 0, \\
-J_{\mathrm{A}(1)} \cdot \partial\left(\beta \mu_{\mathrm{A}}\right) \geq 0, \\
E_{(1) \rho}\left[C_{\mathrm{A}} \beta \mu_{\mathrm{A}} B^{\rho}+\epsilon^{\mu \nu \alpha \rho} \partial_{\mu}\left(\beta H_{\nu}\right) u_{\alpha}\right] \geq 0 .
\end{gathered}
$$

Equations (A20a) and (A20b) are satisfied if we introduce the viscosities and axial conductivity as

$$
\begin{gathered}
T_{(1)}^{\mu \nu}=2 \eta \nabla^{\langle\mu} u_{\perp}^{\nu\rangle}+\zeta \Delta^{\mu \nu} \nabla_{\perp} \cdot u \\
J_{\mathrm{A}(1)}^{\mu}=\lambda_{\mathrm{A}} \nabla_{\perp}^{\mu}\left(\beta \mu_{\mathrm{A}}\right)
\end{gathered}
$$

where $\langle\ldots\rangle$ denotes the symmetric and traceless part, and $\nabla_{\perp}^{\mu} \equiv \Delta^{\mu \nu} \partial_{\nu}$. In general, the viscosities and the diffusion constant $\lambda_{A}$ can be anisotropic, due to the existence of the magnetic field (see Refs. [2-4,25,26] for further discussion and Refs. [43-47] for recent computations of those anisotropic transport coefficients for hot QCD matter).
We now focus on Eq. (A20c), which implies

$$
\left[C_{\mathrm{A}} \beta \mu_{\mathrm{A}} B^{\rho}+\epsilon^{\mu \nu \alpha \rho} \partial_{\mu}\left(\beta H_{\nu}\right) u_{\alpha}\right]=Z^{\rho \nu} E_{(1) \nu}
$$

with a semipositive definite $Z^{\mu \nu}$. To further constrain the form of $Z^{\mu \nu}$, it is convenient to introduce a unit vector in the direction of the magnetic field,

$$
b^{\mu} \equiv \frac{B^{\mu}}{\sqrt{|B|}},
$$

which satisfies $b_{\mu} b^{\mu}=-1$. The spatial projector can be decomposed into the directions parallel and perpendicular to $\boldsymbol{B}$, as

$$
\Delta^{\mu \nu}=-b^{\mu} b^{\nu}+\Delta_{\perp}^{\mu \nu}
$$

where $\Delta_{\perp}^{\mu \nu} \equiv \Delta^{\mu \nu}+b^{\mu} b^{\nu}$. We can introduce two independent dissipative conductivities and the Hall conductivity consistent with the second law of thermodynamics as

$$
Z^{\mu \nu}=-\beta\left[-\sigma_{\|} b^{\mu} b^{\nu}+\sigma_{\perp} \Delta_{\perp}^{\mu \nu}+\sigma_{\mathrm{Hall}} \epsilon^{\mu \nu \alpha \beta} u_{\alpha} b_{\beta}\right]
$$

The reader is referred to Refs. [48-50] for a recent computation of $\sigma_{\|}, \sigma_{\perp}$ for weakly coupled QGP. Note that the term involving the Hall conductivity does not produce entropy. Let us look at the limit where $\sigma_{\|}=\sigma_{\perp} \equiv \sigma$ and $\sigma_{\text {Hall }}=0$, in which case

$$
\left[C_{\mathrm{A}} \mu_{\mathrm{A}} B^{\rho}+\epsilon^{\mu \nu \alpha \rho} \beta^{-1} \partial_{\mu}\left(\beta H_{\nu}\right) u_{\alpha}\right]=-\sigma E_{(1)}^{\rho}
$$

From Eq. (A27), we obtain Eq. (1). The second term on the right in Eq. (A27) represents the $\mathrm{CME}$, as we explained in Sec. II.

\section{APPENDIX B: A DISCUSSION ON VECTOR CHARGE DENSITY AND ELECTRIC FIELD $E^{\mu}$}

In this work, we did not include vector charge density $n$ and electric field $F^{\mu}$ as CMHD variables. As we explained in the Introduction, both $n$ and $E^{\mu}$ are damped out at rates proportional to the electric conductivity $\sigma$. The reason can be understood as follows in this paragraph, where we will discuss the damping of $n$. The case for the electric field is similar. To see the key physics in the simplest way, let us consider the nonrelativistic case. If we substitute Ohm's law $\boldsymbol{j}=\sigma \boldsymbol{E}$ for the current conservation and use the Gauss law $\nabla \cdot \boldsymbol{E}=n$, we obtain

$$
\partial_{t} n=-\sigma n
$$

which indicates that $n$ is damped out with the rate $\sigma$. Instead, the value of $n$ is slaved by the hydrodynamic variables. For example, in Ref. [41], it is found that 
$n=C_{A} \omega \cdot B$ for a chiral fluid in the strong magnetic field limit.

We note in passing that the relativistic Ampere law,

$$
\partial_{\mu} F^{\mu \nu}=J^{\nu}
$$

can be recast into a relaxation equation which tells us that $E^{\mu}$ will approach the expression given by Eq. (1) at a timescale much longer than $1 / \sigma$. To make the argument simple and clear, let us consider a local rest frame, where Eq. (B2) takes the usual form in terms of spatial electric and magnetic fields:

$$
-\frac{\partial \boldsymbol{E}}{\partial t}+\nabla \times \boldsymbol{B}=\boldsymbol{J}=\sigma \boldsymbol{E}+C_{\mathrm{A}} \mu_{\mathrm{A}} \boldsymbol{B},
$$

where we have used Eq. (10). This may be written as

$$
\frac{\partial \boldsymbol{E}}{\partial t}=-\sigma\left[\boldsymbol{E}-\left(\frac{1}{\sigma} \nabla \times \boldsymbol{B}+\frac{C_{\mathrm{A}} \mu_{\mathrm{A}}}{\sigma} \boldsymbol{B}\right)\right] .
$$

What appears inside the bracket on the right-hand side is precisely the first-order constitutive relation for $\boldsymbol{E}$ [cf. Eq. (9)], and the above equation is the relaxation equation of $\boldsymbol{E}$ to its constitutive relation with the relaxation time $1 / \sigma$. This is similar to the Israel-Stewart theory of dissipative hydrodynamics. In the CMHD timescale $(\gg 1 / \sigma)$, the iterations of Eq. (B4) will reduce to the conventional derivative expansions in space-time and $C_{\mathrm{A}}$ that our study is based on, but the microscopic theory is consistent with causality in a way similar to how the IsraelStewart theory restores causality. This suggests that a numerical simulation of CMHD that is consistent with causality may need to use the original Maxwell equations à la Israel-Stewart theory, instead of a finite truncation of derivative expansions.

\section{APPENDIX C: A KUBO FORMULA FOR CHIRAL MAGNETIC CONDUCTIVITY IN CMHD}

In the literature, $\sigma_{\mathrm{A}} \equiv C_{\mathrm{A}} \mu_{A}$ is sometimes referred to as the chiral magnetic conductivity [29]. It is useful to derive a Kubo formula for $\sigma_{\mathrm{A}}$ in CMHD. For this purpose, we consider a fluid at rest, i.e., $u^{\mu}=(1,0,0,0)$, and we replace $\boldsymbol{j}$ in Eq. (10) with $\nabla \times \boldsymbol{H}$. Keeping only the contribution from CME in Eq. (10), we obtain $\boldsymbol{B}=\sigma_{\mathrm{A}}^{-1} \nabla \times \boldsymbol{H}$. Since $\boldsymbol{H}$ is conjugate to $\boldsymbol{B}$, we then have

$$
\sigma_{\mathrm{A}}^{-1}=\lim _{k \rightarrow 0 \omega \rightarrow 0} \frac{1}{k_{l}} \epsilon_{i j k}\left\langle B^{i} B^{j}\right\rangle,
$$

where $\left\langle B^{i} B^{j}\right\rangle$ denotes the retarded Green's function of $\boldsymbol{B}$ in Fourier space. Equation $(\mathrm{C} 1)$ is different from the Kubo formula for $\sigma_{\mathrm{A}}$ in usual cases where magnetic field is considered to be nondynamical (cf. Ref. [29]). Here $\sigma_{\mathrm{A}}$ is related to the retarded Green's function of the magnetic field. The Kubo formula, Eq. (C1), which is new in the literature, opens a possibility to compute $\sigma_{\mathrm{A}}$ in chiral systems in which EM fields are dynamical.

\section{APPENDIX D: ANALYTIC SOLUTIONS OF THE SECULAR EQUATION IN EQ. (14) WITH LIMITING VALUES OF $\theta$}

When $\theta=0$, Eq. (14) reduces to

$$
\left(w-\tilde{c}_{\mathrm{s}}^{2}\right)\left[(w-1)^{2}+\tilde{\epsilon}_{\mathrm{A}}^{2} w\right]=0,
$$

where $\tilde{\epsilon}_{\mathrm{A}}=\epsilon_{\mathrm{A}} / u_{\mathrm{A}}, w=V / u_{A}$, and we find the six solutions as

$$
V= \pm c_{\mathrm{s}}, \quad V= \pm\left(\sqrt{u_{\mathrm{A}}^{2}-\frac{\epsilon_{\mathrm{A}}^{2}}{4}} \pm i \frac{\epsilon_{\mathrm{A}}}{2}\right)
$$

where the signs are taken for all combinations. The first two are ordinary sound modes without any modification by the magnetic field or anomaly, since the pressure of the magnetic field does not contribute to that along the wave vector. The remaining four modes arise as the results of the mixing between the Alfvén and magnetosonic waves, where the $V$ 's are complex when $\epsilon_{\mathrm{A}}^{2}<4 u_{\mathrm{A}}^{2}$, and are pure imaginary when $\epsilon_{\mathrm{A}}^{2}>4 u_{\mathrm{A}}^{2}$. Those regions correspond to Phases IIA and IIB, respectively. In both phases, positive and negative imaginary parts appear in pairs, and the signs depend on that of $\epsilon_{\mathrm{A}}$.

When $\theta= \pm \pi / 2$, the secular equation reduces to

$$
w\left[w^{2}-w\left(1+\tilde{c}_{\mathrm{s}}^{2}-\tilde{\epsilon}_{\mathrm{A}}^{2}\right)-\left(\tilde{\epsilon}_{\mathrm{A}} \tilde{c}_{\mathrm{s}}\right)^{2}\right]=0,
$$

and we find the six solutions as

$$
V=0, \quad V= \pm \sqrt{\kappa \pm \sqrt{\kappa^{2}+\nu}}
$$

where $\kappa=\left(u_{\mathrm{A}}^{2}+c_{\mathrm{S}}^{2}-\epsilon_{\mathrm{A}}^{2}\right)$ and $\nu=\left(\epsilon_{\mathrm{A}} c_{\mathrm{S}}\right)^{2}$. The presence of two vanishing solutions means that group velocity becomes zero. There are two real solutions, which provide the waves propagating in the opposite directions. Their velocities are modified by the anomaly effect. The remaining two solutions are vanishing in the absence of anomaly effects $(\nu=0)$, which, however, become a pair of positive and negative pure imaginary numbers when $\nu \neq 0$. They do not propagate, but grow or dissipate exponentially in time, respectively. 
[1] L. Landau and E. Lifshitz, Fluid Mechanics (Pergamon Press, New York, 1987).

[2] P. Kovtun, J. High Energy Phys. 07 (2016) 028.

[3] S. Grozdanov, D. M. Hofman, and N. Iqbal, Phys. Rev. D 95, 096003 (2017).

[4] J. Hernandez and P. Kovtun, J. High Energy Phys. 05 (2017) 001.

[5] N. Yamamoto, Phys. Rev. D 93, 125016 (2016).

[6] I. Rogachevskii, O. Ruchayskiy, A. Boyarsky, J. Frhlich, N. Kleeorin, A. Brandenburg, and J. Schober, Astrophys. J. 846, 153 (2017).

[7] A. Boyarsky, J. Frohlich, and O. Ruchayskiy, Phys. Rev. D 92, 043004 (2015).

[8] M. Giovannini, Phys. Rev. D 94, 081301 (2016).

[9] D. E. Kharzeev, L. D. McLerran, and H. J. Warringa, Nucl. Phys. A803, 227 (2008).

[10] D. E. Kharzeev, J. Liao, S. A. Voloshin, and G. Wang, Prog. Part. Nucl. Phys. 88, 1 (2016).

[11] Q. Li, D. E. Kharzeev, C. Zhang, Y. Huang, I. Pletikosic, A. V. Fedorov, R. D. Zhong, J. A. Schneeloch, G. D. Gu, and T. Valla, Nat. Phys. 12, 550 (2016).

[12] J. Xiong, S. K. Kushwaha, T. Liang, J. W. Krizan, M. Hirschberger, W. Wang, R. J. Cava, and N. P. Ong, Science 350, 413 (2015).

[13] M. Joyce and M. E. Shaposhnikov, Phys. Rev. Lett. 79, 1193 (1997).

[14] A. Boyarsky, J. Fröhlich, and O. Ruchayskiy, Phys. Rev. Lett. 108, 031301 (2012).

[15] M. Stephanov and Y. Yin, Phys. Rev. D 98, 036006 (2018).

[16] H. Nielsen and M. Ninomiya, Phys. Lett. B 130, 389 (1983).

[17] A. Vilenkin, Phys. Rev. D 22, 3080 (1980).

[18] K. Fukushima, D. E. Kharzeev, and H. J. Warringa, Phys. Rev. D 78, 074033 (2008).

[19] D. E. Kharzeev, K. Landsteiner, A. Schmitt, and H.-U. Yee, Lect. Notes Phys. 871, 1 (2013).

[20] D. E. Kharzeev, Prog. Part. Nucl. Phys. 75, 133 (2014).

[21] V. A. Miransky and I. A. Shovkovy, Phys. Rep. 576, 1 (2015).

[22] K. Hattori and X.-G. Huang, Nucl. Sci. Tech. 28, 26 (2017).

[23] Y. Hirono, D. E. Kharzeev, and Y. Yin, Phys. Rev. Lett. 117, 172301 (2016).

[24] W. Israel, Gen. Relativ. Gravit. 9, 451 (1978).
[25] X.-G. Huang, M. Huang, D. H. Rischke, and A. Sedrakian, Phys. Rev. D 81, 045015 (2010).

[26] X.-G. Huang, A. Sedrakian, and D. H. Rischke, Ann. Phys. (Amsterdam) 326, 3075 (2011).

[27] D. T. Son and P. Surowka, Phys. Rev. Lett. 103, 191601 (2009).

[28] D.-F. Hou, H. Liu, and H.-c. Ren, Phys. Rev. D 86, 121703 (2012).

[29] D. E. Kharzeev and H. J. Warringa, Phys. Rev. D 80, 034028 (2009).

[30] P. Hosur and X. Qi, C.R. Phys. 14, 857 (2013).

[31] S. J. Brodsky and P. Hoyer, Phys. Rev. D 83, 045026 (2011).

[32] J. Jackson, Classical Electrodynamics (Wiley, New York, 1975).

[33] D. Biskamp, Nonlinear Magnetohydrodynamics (Cambridge University Press, Cambridge, England, 1997), Vol. 1.

[34] M. Laine, J. High Energy Phys. 10 (2005) 056.

[35] Y. Akamatsu and N. Yamamoto, Phys. Rev. Lett. 111, 052002 (2013).

[36] Y. Hirono, D. Kharzeev, and Y. Yin, Phys. Rev. D 92, 125031 (2015).

[37] G. M. Newman, J. High Energy Phys. 01 (2006) 158.

[38] D. E. Kharzeev and H.-U. Yee, Phys. Rev. D 83, 085007 (2011).

[39] Y. Jiang, X.-G. Huang, and J. Liao, Phys. Rev. D 92, 071501 (2015).

[40] N. Yamamoto, Phys. Rev. Lett. 115, 141601 (2015).

[41] K. Hattori and Y. Yin, Phys. Rev. Lett. 117, 152002 (2016).

[42] V. Galitski, M. Kargarian, and S. Syzranov, Phys. Rev. Lett. 121, 176603 (2018).

[43] K. Tuchin, J. Phys. G 39, 025010 (2012).

[44] K. Fukushima, K. Hattori, H.-U. Yee, and Y. Yin, Phys. Rev. D 93, 074028 (2016).

[45] S. Li and H.-U. Yee, Phys. Rev. D 97, 056024 (2018).

[46] S. I. Finazzo, R. Critelli, R. Rougemont, and J. Noronha, Phys. Rev. D 94, 054020 (2016); 96, 019903(E) (2017).

[47] K. Hattori, X.-G. Huang, D. H. Rischke, and D. Satow, Phys. Rev. D 96, 094009 (2017).

[48] K. Hattori, S. Li, D. Satow, and H.-U. Yee, Phys. Rev. D 95, 076008 (2017).

[49] K. Hattori and D. Satow, Phys. Rev. D 94, 114032 (2016).

[50] K. Fukushima and Y. Hidaka, Phys. Rev. Lett. 120, 162301 (2018). 